Check for updates

Cite this: RSC Adv., 2021, 11, 1430

Received 16th October 2020

Accepted 9th December 2020

DOI: $10.1039 / \mathrm{d}$ Ora08826a

rsc.li/rsc-advances

\section{Three-dimensional, symmetrically assembled microfluidic device for lipid nanoparticle production $\dagger$}

\author{
Niko Kimura, (D) $\dot{t}^{a}$ Masatoshi Maeki, (D) $\ddagger^{* b c}$ Kosuke Sasaki, ${ }^{d}$ Yusuke Sato, ${ }^{d}$ \\ Akihiko Ishida, id b Hirofumi Tani, iD b Hideyoshi Harashima ${ }^{\mathrm{d}}$ \\ and Manabu Tokeshi (iD *bef
}

Sub $100 \mathrm{~nm}$-sized lipid nanoparticles (LNPs) have been widely used in drug delivery systems (DDSs). The size of the LNPs is an important parameter for the DDS performance, such as biodistribution and gene silencing using siRNAs. However, the LNPs prepared by the conventional preparation method show a wide size distribution. To improve the LNP size distribution, we developed a microfluidic device, named the iLiNPTM device, in a previous study. This device could produce LNPs in the size range of 20 to $150 \mathrm{~nm}$, but the size distribution of the large-sized LNPs needs to be further improved. From the viewpoint of the LNP formation process, a homogeneous and slow rate dilution of ethanol plays an important role in improving the large-size LNP size distribution. In this study, we developed a three-dimensional, symmetrically assembled microfluidic device named the 3D-iLiNP device with the aim of precise size control of large-sized LNPs. We designed the 3D-iLiNP device using a computational fluid dynamics simulation and demonstrated that the 3D-iLiNP device can improve the LNP size distribution. The gene silencing activity of four kinds of siRNA-loaded LNPs was investigated via in vitro and in vivo experiments to elucidate the effect of the LNP size distribution. The results revealed that the LNPs with a size between 90 and $120 \mathrm{~nm}$ showed higher gene silencing activity than those with other sizes. The 3D-iLiNP device is expected to improve DDS performance by precisely controlling the size of LNPs.

\section{Introduction}

Lipid nanoparticle (LNP)-based drug delivery systems (DDSs) are among the most advanced nanomedicine systems. The size of LNPs plays an important role in both the LNP biodistribution and LNP performance, such as antitumor effect and gene silencing activity. ${ }^{1-7}$ Generally, small-sized LNPs are desirable for stromal rich tumor tissues, such as pancreatic cancer. On

${ }^{a}$ Graduate School of Chemical Sciences and Engineering, Hokkaido University, Kita 13 Nishi 8, Kita-ku, Sapporo, 060-8628, Japan. Fax: +81-11-706-6745; Tel: +81-11-7066744

${ }^{b}$ Division of Applied Chemistry, Faculty of Engineering, Hokkaido University, Kita 13 Nishi 8, Kita-ku, Sapporo 060-8628, Japan. E-mail: tokeshi@eng.hokudai.ac.jp; m. maeki@eng.hokudai.ac.jp; Fax: +81-11-706-6745; Tel: +81-11-706-6745

'JST PRESTO, 4-1-8 Honcho, Kawaguchi, Saitama, 332-0012, Japan

${ }^{d}$ Faculty of Pharmaceutical Sciences, Hokkaido University, Kita 12 Nishi 8, Kita-ku, Sapporo 060-0812, Japan

'Innovative Research Center for Preventive Medical Engineering, Nagoya University, Furo-cho, Chikusa-ku, Nagoya 464-8601, Japan

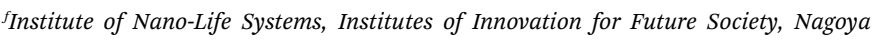
University, Furo-cho, Chikusa-ku, Nagoya 464-8601, Japan

$\dagger$ Electronic supplementary information (ESI) available. See DOI: $10.1039 /$ d0ra08826a

$\$ N$. K. and M. M. contributed equally to this work. the other hand, $100 \mathrm{~nm}$-sized LNPs can deliver drugs or RNA into the hepatocytes of liver tissues passing through the fenestrae (with an approximate diameter of 100-150 nm). ${ }^{8}$ Recently, the relationship between the LNP size and DDS performance has been investigated widely (Table S1 $\dagger$ ). In previous studies, this relationship was investigated with LNPs with a size of 10$50 \mathrm{~nm}$. However, LNPs are produced with a size distribution in spite of size tuning such as extrusion using a polycarbonate filter and ultra-sonication. ${ }^{1,9}$ Therefore, a precise LNP size control method is indispensable for understanding the effect of the LNP size on the DDS performance.

Solvent injection using microfluidic devices can control the LNP size more precisely compared to conventional batch scale production and size tuning methods. ${ }^{\mathbf{1 0 - 1 3}}$ In the microfluidicbased LNP production method, the LNP size was controlled by the ethanol dilution rate. Therefore, the flow conditions, such as the total flow rate and flow rate ratio (FRR) of aqueous phase to lipid phase, and the microchannel geometry are key parameters for controlling the LNP size. ${ }^{13-16}$ To control the LNP size, the LNP formation behavior has been investigated using several types of microfluidic devices (Table S2†). The microfluidic hydrodynamic focusing device ${ }^{17}$ and the chaotic micromixer device ${ }^{15}$ are typical microfluidic devices for the production of LNPs with sizes of 50-60 and 30-60 nm, 
respectively. Although these LNPs are smaller than those produced with conventional methods, they do not cover the size range summarized in Table S1. $\dagger$ To address the LNP size controllability, we developed a microfluidic device named iLiNP ${ }^{\text {TM }}$ (Fig. 1, 2D-iLiNP device). ${ }^{\mathbf{1 4}}$ The iLiNP device was equipped with baffle structures and induced a generation of secondary flow at high total flow rate conditions. As a result, the iLiNP device achieved the production of LNPs in the average size range from 20 to $150 \mathrm{~nm}$. However, the size distribution of large-sized LNPs needs to be improved to understand the influence of the LNP size on the DDS performance in detail.

Theoretically, the dilution rate and mixing homogeneity affect the LNP size and size distribution, respectively. Therefore, the chaotic micromixer device, which induces chaotic advection in the microchannel, allows the production of homogeneoussized LNPs. In contrast, the 2D-iLiNP device allows the production of small- and large-size LNPs in the high and low flow rate conditions, respectively; the large-sized LNPs are formed through diffusion-based ethanol dilution. However, although fluid control is crucial for the improvement of the LNP size distribution, in particular for large-sized LNPs, the homogeneous and slow rate dilution is still challenging. From the viewpoint of fluid dynamics and mass transportation, the homogeneous and slow rate dilution is a competitive phenomenon to diffusion. To the best of our knowledge, the microfluidic device to achieve homogeneous and slow rate

(A)
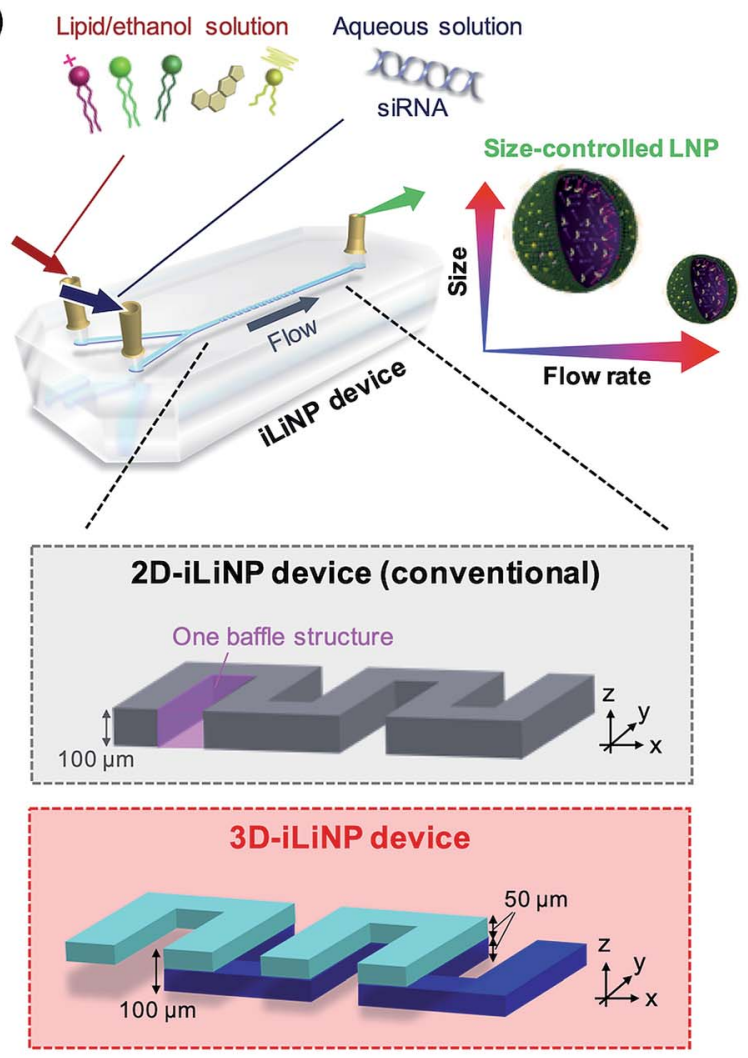

dilution for the improvement of the LNP size distribution has not been reported.

In this paper, we report a three-dimensional (3D), symmetrically assembled microchannel, named the 3D-iLiNP device, based on the 2D-iLiNP device. We designed the 3D baffle structures using computational fluid dynamics (CFD) simulations. We demonstrated the homogeneous and slow rate ethanol dilution by the generation of 3D secondary flows at the low flow rate condition. Finally, we investigated the effect of the LNP size distribution on the gene silencing activity via in vitro and in vivo experiments using siRNA-loaded LNPs.

\section{Results and discussion}

\section{Design of the 3D-iLiNP device through computational fluid dynamics (CFD) simulations}

Fig. 1 shows a schematic of the 3D-iLiNP device equipped with 20 baffle structures based on the 2D-iLiNP device. ${ }^{14}$ With the 3D-iLiNP device, we predicted a secondary flow in the $x-z$ direction in addition to that in the $y-z$ direction at the $3 \mathrm{D}$-baffle structures; this secondary flow would allow the acceleration of the ethanol dilution of the lipid solution. Fig. 2(A) shows the 3D views, cross-sectional views, and CFD results of the $2 \mathrm{D}$ - and $3 \mathrm{D}$ iLiNP devices. As a proof of the concept experiment, we preliminarily designed the three-types of the 3D-iLiNP devices. The 3D-iLiNP devices consisted of top and bottom layers with different channel thickness; (a) 50-50 $\mathrm{m}$, (b) 80-20 $\mu \mathrm{m}$, and (c)

(B)
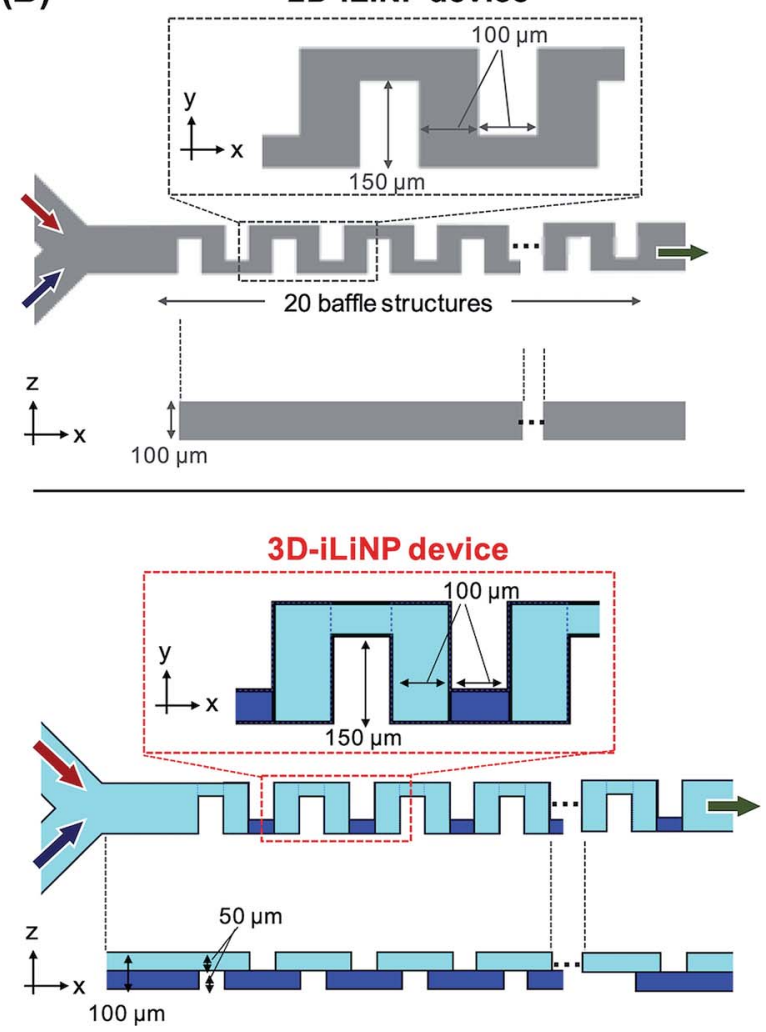

Fig. 1 (A) Schematic and (B) top and cross-sectional views of the 2D- and 3D-iLiNP devices. 


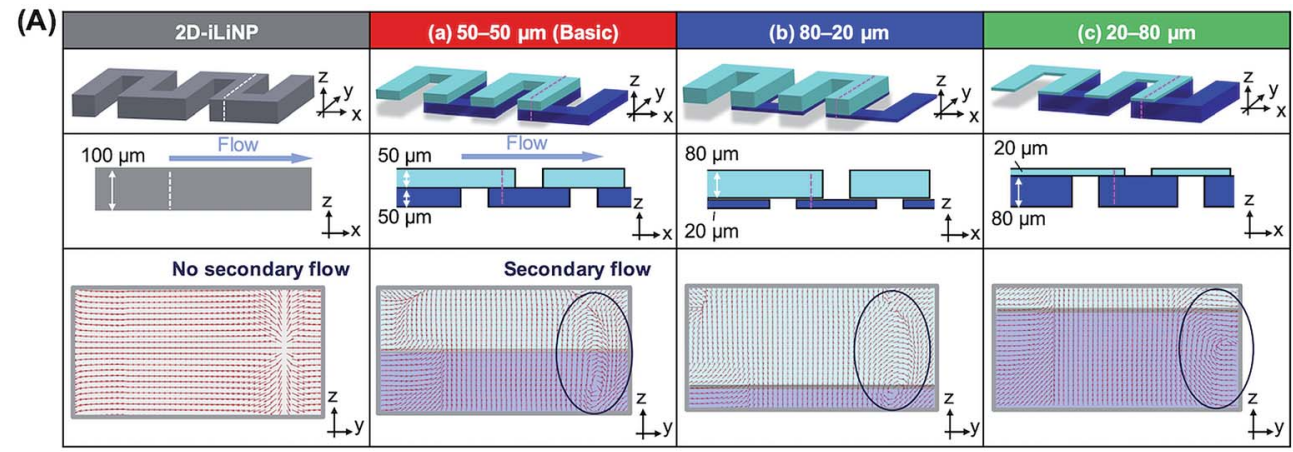

(B)
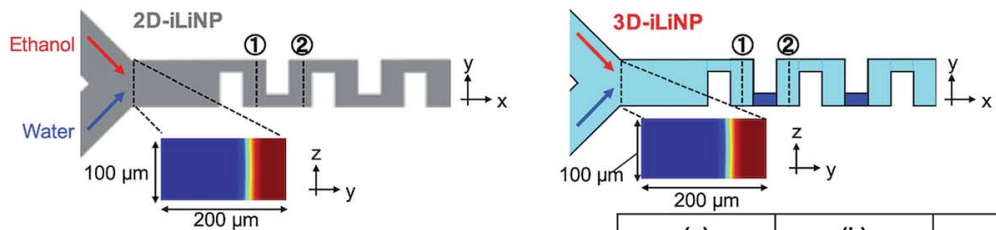

Ethanol concentration
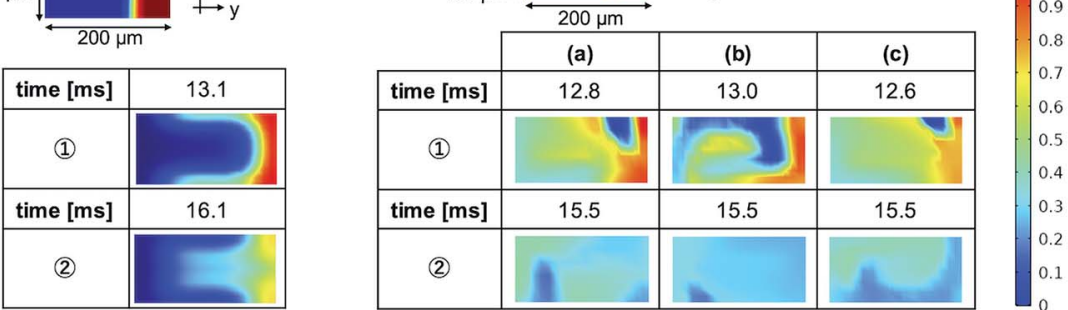

(C)

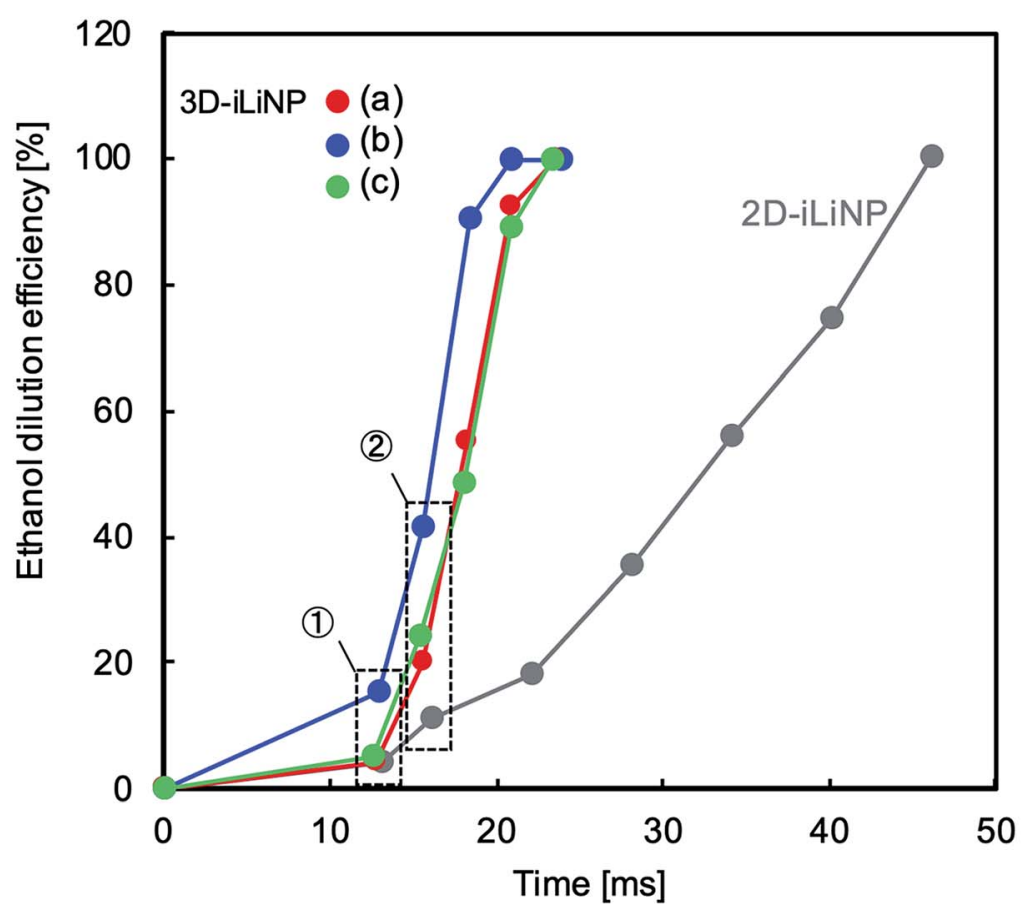

Fig. 2 CFD simulation of ethanol dilution in the 2D- and 3D-iLiNP devices. The total flow rate was $50 \mu \mathrm{L} \mathrm{min}{ }^{-1}$ and the FRR was 3. (A) Streamlines at the cross-section of the 2D- and 3D-iLiNP devices. The dashed line region in the 3D views represent the cross-section of the CFD results. The 3D-iLiNP devices consisted of top and bottom layers with different channel thickness; (a) 50-50 $\mu \mathrm{m}$, (b) $80-20 \mu \mathrm{m}$, and (c) 20-80 $\mu \mathrm{m}$. (B) Comparison of ethanol dilution process of the 2D- and 3D-iLiNP devices. (C) Ethanol dilution efficiency of the 2D-and 3D-iLiNP devices.

20-80 $\mu \mathrm{m}$. The total flow rate and the FRR (water to ethanol) was set to $50 \mu \mathrm{L} \mathrm{min} \operatorname{mos}^{-1}$ and 3 , respectively. We focused on the generation of the secondary flow in the 3D-baffle structures. As expected, the secondary circulation flow was generated at the baffle structures of the 3D-iLiNP devices, whereas no secondary flow was observed in the 2D-iLiNP device. Fig. 2(B) and (C) shows a comparison of the ethanol dilution process and ethanol dilution efficiency of the 2D- and 3D-iLiNP devices. The time 
differences to reach the solutions to cross-sections were shorter than $0.6 \mathrm{~ms}$. In this FRR condition, the final ethanol concentration was $25 \%$. The $2 \mathrm{D}$-iLiNP device retained a high concentration of ethanol at the corner of the microchannel $16 \mathrm{~ms}$ after injection. The 3D-iLiNP devices diluted ethanol homogeneously compared with the 2D-iLiNP device. The 3D-iLiNP devices showed complete ethanol dilution within $20 \mathrm{~ms}$, regardless of the device design (Fig. 2(C)). The design of the basic-type, 80-20 $\mu \mathrm{m}$, and $20-80 \mu \mathrm{m} 3 \mathrm{D}$-iLiNP devices did not significantly affect the dilution performance of ethanol in the CFD simulation. The results of the CFD simulation confirm that the 3D-iLiNP device at a low total flow rate is a more suitable structure for homogeneous and slow rate ethanol dilution compared with the $2 \mathrm{D}$ iLiNP device. ${ }^{14,15}$ This fluid dynamics is significant to control the LNP size at the low flow rate condition. Therefore, we carried out the evaluation of the LNP size controllability of the 3D-iLiNP devices.

\section{Evaluation of the LNP size controllability of the 3D-iLiNP devices}

We determined the 3D-iLiNP device design with the highest LNP size controllability. Fig. 3(A) shows the size distribution of 1-palmitoyl-2-oleoyl-sn-glycero-3-phosphocholine (POPC) LNPs produced using the $2 \mathrm{D}$ and $3 \mathrm{D}$-iLiNP devices, the basic-type, 80$20 \mu \mathrm{m}$, and $20-80 \mu \mathrm{m}$, at a total flow rate and FRR of 50 $\mu \mathrm{L} \min ^{-1}$ and 3 , respectively. The basic-type 3D-iLiNP device showed the narrowest LNP size distribution among the four devices, the 2D and three types of 3D-iLiNP devices. Fig. 3(B) shows the average LNP size and coefficient variation (CV) value and Fig. 3(C) shows the polydispersity index (PDI) of the LNPs produced with the four devices. The average sizes of the POPC
LNPs produced with the basic-type, $80-20 \mu \mathrm{m}$, and $20-80 \mu \mathrm{m}$ 3D-iLiNP devices were 101, 78, and $141 \mathrm{~nm}$, respectively. The CV values and PDI were approximately $7-12 \%$ and $0.17-0.23$, respectively. In comparison with the $2 \mathrm{D}$-iLiNP device, the basictype 3D-iLiNP device showed small CV value and PDI even with a large particle size of approximately $100 \mathrm{~nm}$. We assume the sharp change of the device geometry affects the fluid stability and LNP size controllability. From the viewpoint of microfluidics, two solutions introduce into a narrow microchannel is unstable compared with that of a wide microchannel due to the backpressure and the pressure balance of two solutions. As a result, the basic-type $3 \mathrm{D}$-iLiNP device showed small $\mathrm{CV}$ value and PDI value. The relationship between the flow condition and the average LNP size is summarized in Fig. S2(A). $\dagger$ We also measured the concentrations of LNPs using a nanoparticle tracking analyzer (NS300, NanoSight, Malvern Instruments, Worcestershire, UK) (Fig. S2(B) $\dagger$ ). The basic-type 3D-iLiNP device clearly improved the LNP size distribution. From these results, we decided to use the basic-type 3D-iLiNP device in this study.

Fig. 4 shows the size distributions of the LNPs produced with the 2D- and 3D-iLiNP devices at total flow rates of 50 or 500 $\mu \mathrm{L} \min ^{-1}$ and FRRs of 3 and 9. In a previous study, it was reported that the increase in the total flow rate and FRR induces the production of small-sized LNPs. ${ }^{14}$ From Fig. 4, it can be seen that the size distribution of the LNPs produced using the 3DiLiNP device in all flow conditions was significantly more homogeneous than that of the LNP produced with the 2D-iLiNP device. In particular, a single narrow peak can be observed in the size distribution of the LNPs produced with the 3D-iLiNP device at the flow rate of $50 \mu \mathrm{L} \mathrm{min}{ }^{-1}$ and FRR of 3. Fig. 5(A) and (B) show a summary of the size of the LNPs produced with
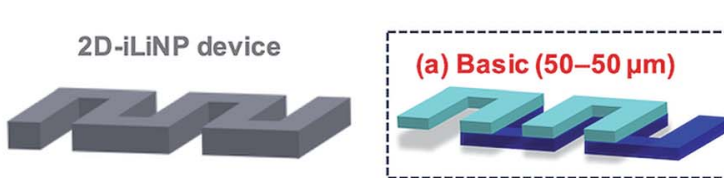

3D-iLiNP devices

(b) $80-20 \mu \mathrm{m}$

(B)

(A)

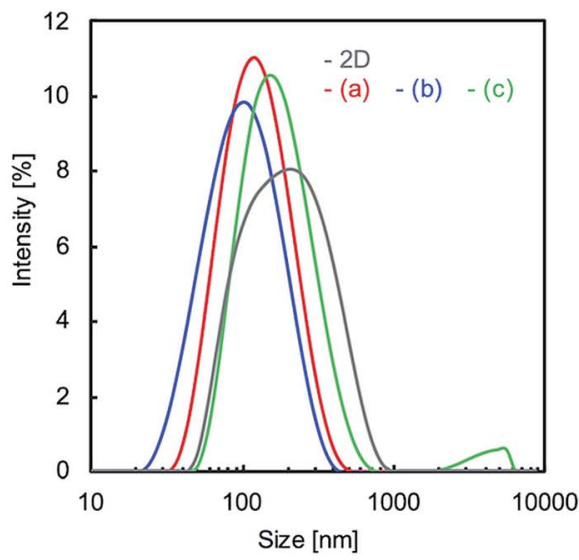

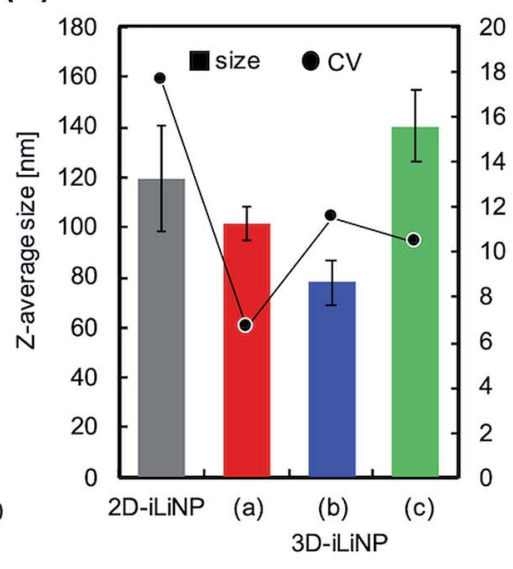

(C)

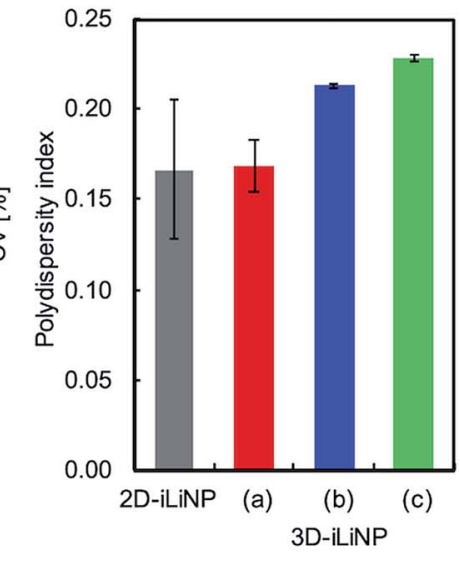

Fig. 3 (A) Size distributions of the POPC LNPs prepared with the 2D- (gray) and 3D-iLiNP devices. The total flow rate was $50 \mu \mathrm{L}$ min ${ }^{-1}$ and the FRR was 3. Red, blue, and green represent the (a) basic, (b) 80-20 $\mu \mathrm{m}$, and (c) 20-80 $\mu \mathrm{m}$ devices, respectively. (B) Average sizes and coefficient variation (CV) values of the POPC LNPs. (C) Average values of the polydispersity index (PDI) of the POPC LNPS. 
(A)

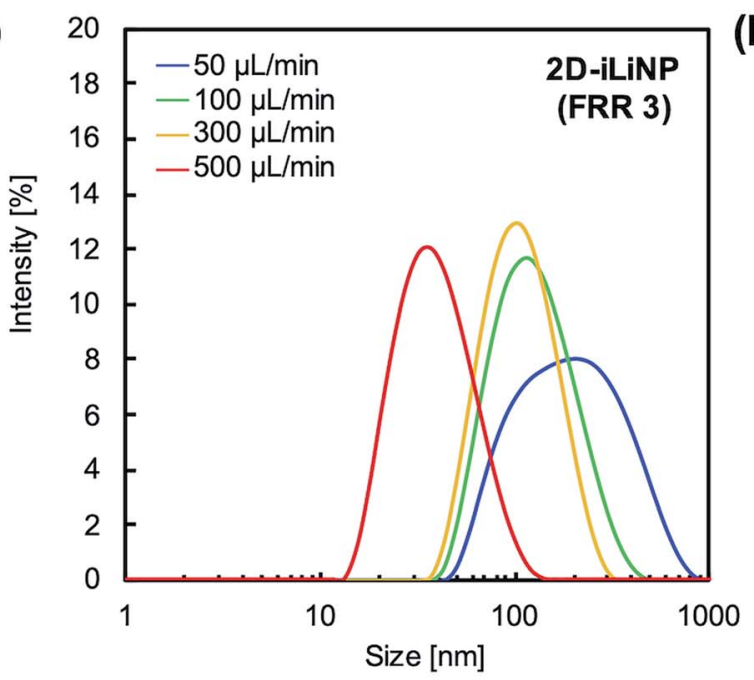

(C)

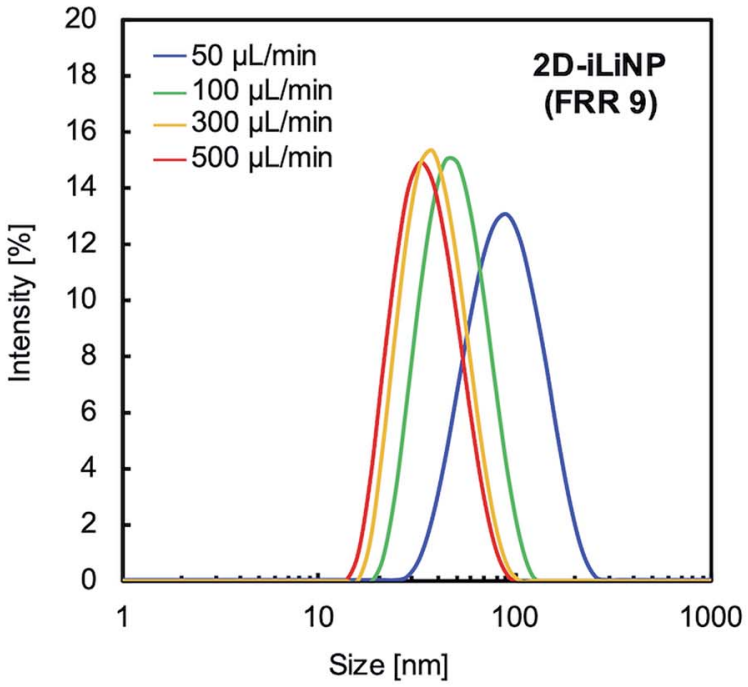

(B)
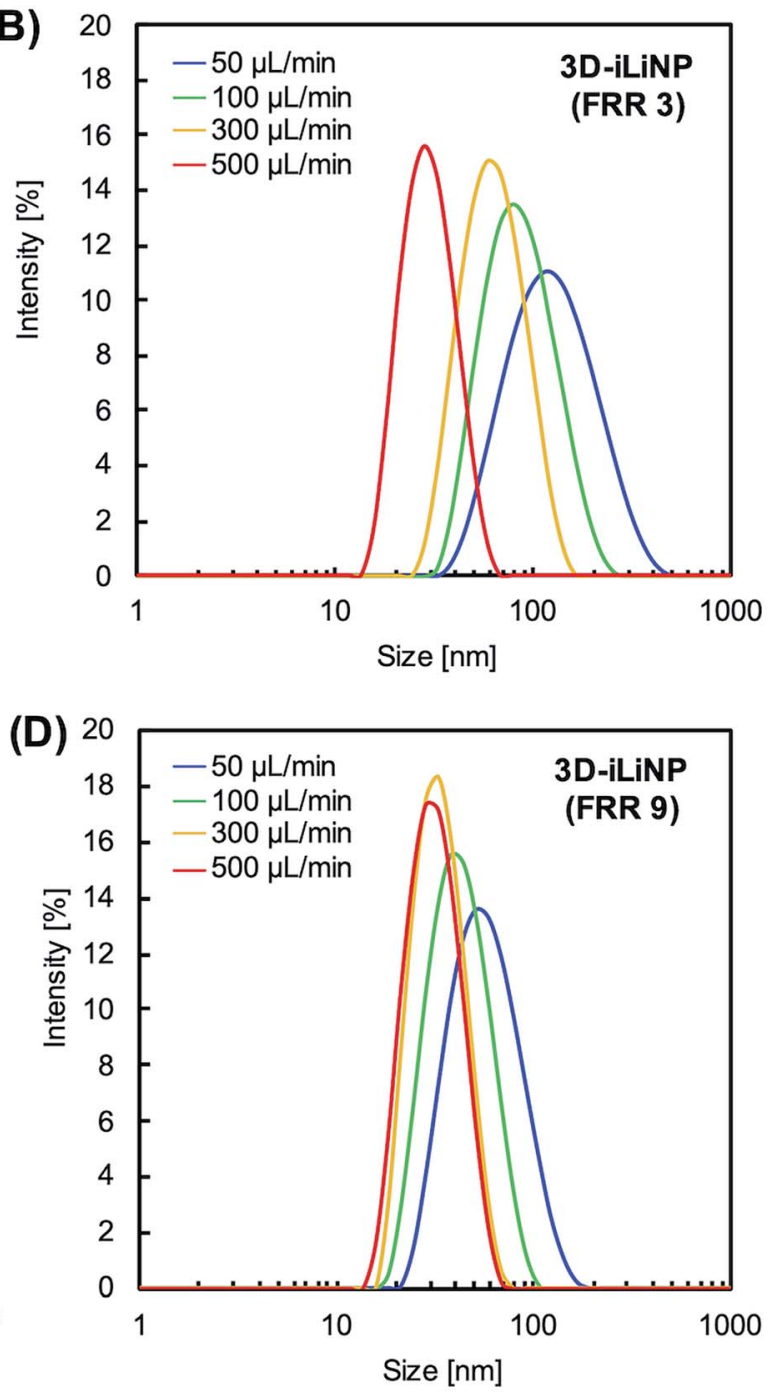

Fig. 4 Size distributions of the POPC LNPs prepared at FRRs of (A and B) 3 and (C and D) 9. (A) and (C) 2D-iLiNP device; (B) and (D) 3D-iLiNP device.

the 2D- and 3D-iLiNP devices with an FRR of 3 and 9, respectively. With an FRR of 3, the LNP size range of the iLiNP devices was approximately $30-100 \mathrm{~nm}$, and the LNPs produced with the 3D-iLiNP device were slightly smaller than those produced with the 2D-iLiNP device. With an FRR of 9, the LNP size had a similar trend to that observed at an FRR of 3, and the size range of the LNPs produced with 3D-iLiNP device was 20$50 \mathrm{~nm}$. By controlling the fluid flow, the size of the LNPs produced with the 3D-iLiNP device could be controlled with $10 \mathrm{~nm}$ precision (Fig. S3†), even when the device design was modified for mass production (Fig. S4 $\dagger$ ). Both the 2D- and 3DiLiNP devices allowed the control of the LNP size through the control of the flow conditions; however, the 3D-iLiNP device led to LNPs with lower CV value in the low flow condition (Fig. 5(C) and (D)). In this study, we decide to $10 \%$ value of $\mathrm{CV}$ (for example, $20 \mathrm{~nm} \pm 2 \mathrm{~nm}$ and $100 \mathrm{~nm} \pm 10 \mathrm{~nm}$ ) is a benchmark to evaluate the LNP size controllability. ${ }^{16}$ At all total flow values, the $\mathrm{CV}$ value of the LNPs produced with the 3D-iLiNP device was at least $7 \%$ smaller than that of the LNPs produced with the $2 \mathrm{D}$ iLiNP device.

In the microfluidic-based LNP preparation method, the dilution rate affects the LNP size and the mixing homogeneity affects the LNP size distribution. ${ }^{15}$ From the viewpoint of fluid dynamics and mass transportation, it is difficult to achieve a homogeneous distribution and slow dilution rate because the homogeneous and slow rate dilution is a competitive phenomenon to diffusion. The concentration gradient induced by diffusion is the major reason for the formation of heterogeneous-sized LNPs because ethanol dilution is a trigger for LNP formation. Therefore, a microfluidic device suitable for low flow rate conditions is indispensable for producing homogeneous- and large-sized LNPs. On the other hand, a homogeneous size distribution and rapid dilution can be achieved with several types of microfluidic devices, such as the chaotic micromixer and 2D-iLiNP devices. The chaotic micromixer device allows homogeneous mixing under low flow rates; $;^{18}$ 
(A)

however, it could not produce the large-sized LNPs (Table S2 $\dagger$ ). ${ }^{15}$ In the case of the 2D-iLiNP device, the formation of the secondary flow plays an important role in the LNP size controllability and depends on the total flow rate. ${ }^{14}$ The 2DiLiNP device allows the control of the LNP Z-average size in the wide range of $20-120 \mathrm{~nm}$ through the flow condition. However, the formation of the secondary flow at the low flow rate condition in the $2 \mathrm{D}$-iLiNP was not sufficient to produce homogeneous- and large-sized LNPs. In this study, we revealed that the homogeneous and slow rate dilution was compatible with the rapid dilution by the 3D-iLiNP device, which allowed the production of LNPs in the size range of $20-100 \mathrm{~nm}$ with low $\mathrm{CV}$ values.

\section{Application for siRNA-LNP systems}

We applied the 3D-iLiNP device for siRNA-encapsulated LNP production to investigate the effect of the LNP size distribution on the gene silencing activity in in vitro and in vivo experiments. Our original synthesized $\mathrm{pH}$-sensitive cationic lipid, named CL4H6, ${ }^{19}$ and 1,2-dioleoyl-3-dimethylammonium-
(B)

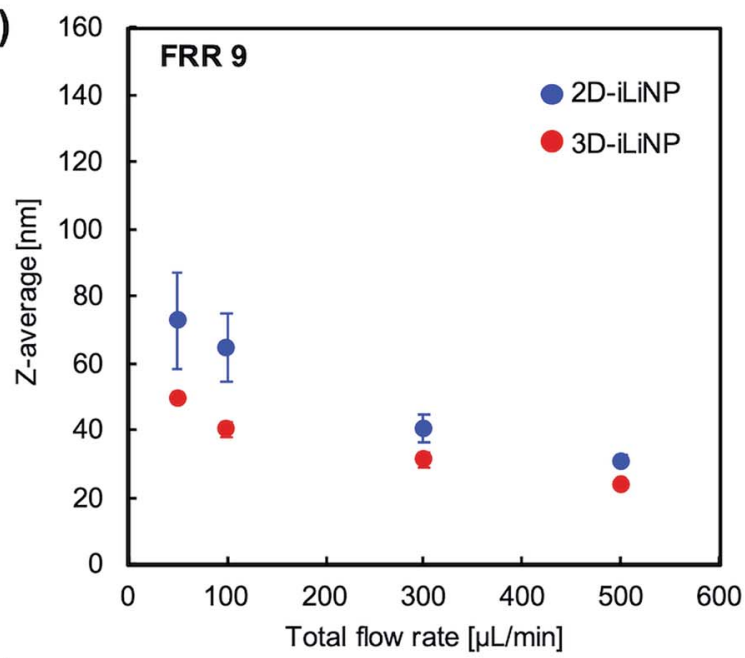

(D)

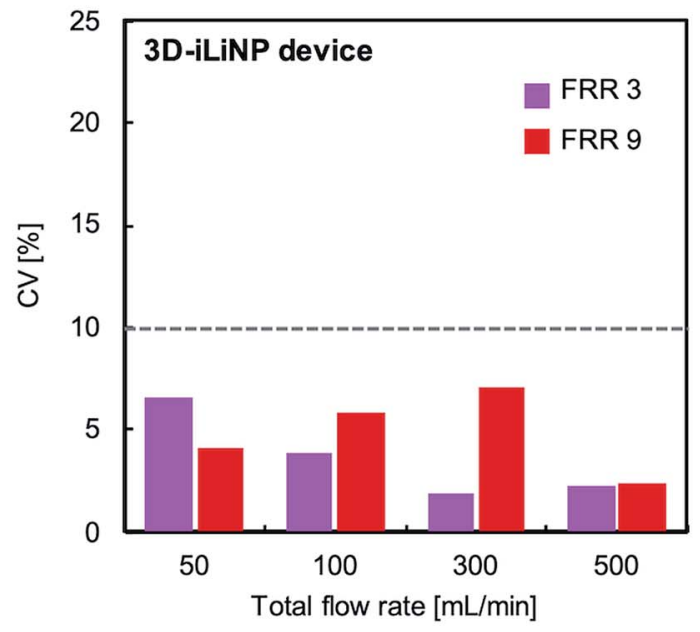

Fig. 5 Average LNP size as a function of the total flow rate with an FRR of (A) 3 and (B) 9. (C, D) CV values of the LNPs prepared with the 2D- and 3D-iLiNP devices, respectively.

propane (DODAP), a commercially available lipid, were used as the main components of the lipid systems. Fig. 6(A) shows a schematic of the CL4H6-based LNP preparation process and in vivo experimental design. At total flow rates of 50 or 500 $\mu \mathrm{L} \min ^{-1}$, the 3D-iLiNP device produced 99 and $59 \mathrm{~nm}$ LNPs (Fig. 6(B)). At the same total flow rates, the average size of the CL4H6 LNPs produced with the 2D-iLiNP device were 102 and $69 \mathrm{~nm}$, respectively. The siRNA encapsulation efficiency of the LNPs was higher than $97 \%$ (Table S3 $\dagger$ ). The CV values of the LNPs produced with the 3D-iLiNP device were lower than 10\%, while the PDI was almost the same among the four types of LNPs. We did not observe a statistically significant difference on the average size of the 99 and $102 \mathrm{~nm}$-sized LNPs. To investigate the effect of the LNP size distribution on the gene silencing activity, we classified the LNP size into five size ranges based on the LNP size distribution (Fig. 6(C) and S5 †): $<40,40-60,60-90,90-120$, and $>120 \mathrm{~nm}$. From the LNP size distribution, we confirmed that the $40-60$ and $90-120 \mathrm{~nm}$ LNPs were the main size ranges of the 59 and $99 \mathrm{~nm}$ sized LNPs prepared with the 3D-iLiNP device, respectively. The 
(A)

10 mM CL4H6/DSPC/cholesterol/DMG-PEG2K (50/10/38.5/1.5 mol\%)

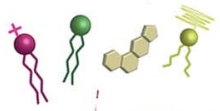

siRNAs in $10 \mathrm{mM}$ citrate buffer solution (pH3.0)
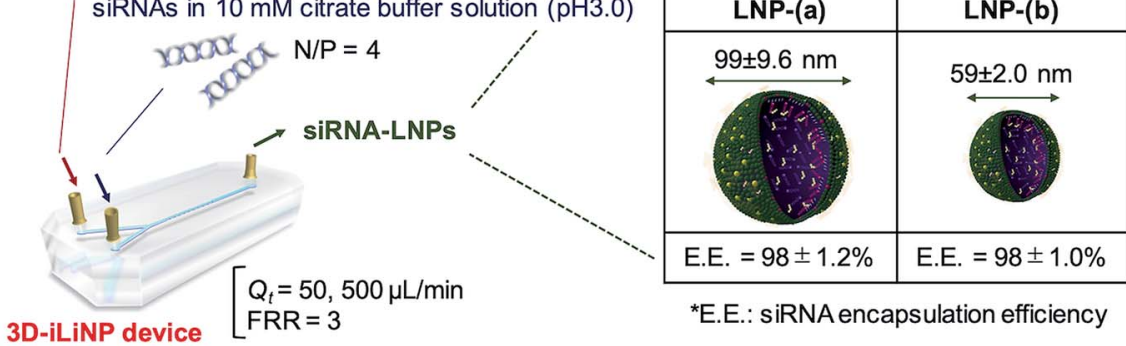

SIRNA-LNPs

\begin{tabular}{c|c|}
$\begin{array}{c}\text { Total flow rate } \\
=50 \mu \mathrm{L} / \mathrm{min}\end{array}$ & $\begin{array}{c}\text { Total flow rate } \\
=500 \mu \mathrm{L} / \mathrm{min}\end{array}$ \\
\hline LNP-(a) & LNP-(b) \\
\hline $99 \pm 9.6 \mathrm{~nm}$ & \\
\hline & \\
\hline
\end{tabular}

${ }^{\star}$ E.E.: siRNA encapsulation efficiency

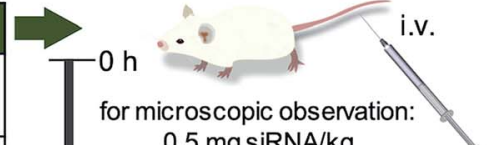

$0.5 \mathrm{mg}$ siRNA $/ \mathrm{kg}$

for gene expression assay: $0.1 \mathrm{mg}$ siRNA/kg

$-1 \mathrm{~h}$

Liver collected

$\rightarrow$ Microscopic observation

$\sqrt{ } 24$

FVII gene-expression assay
(B)

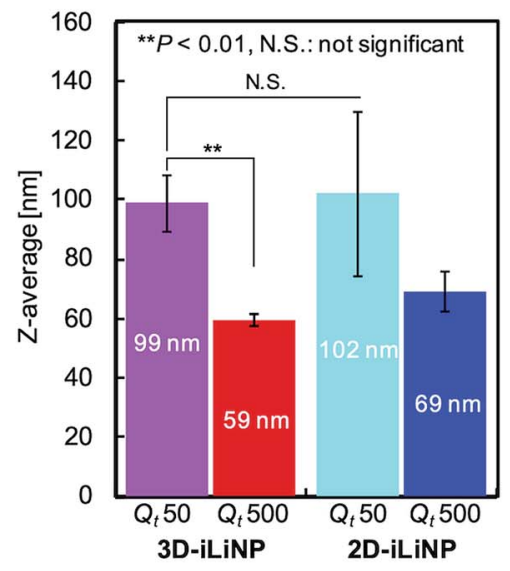

(C)

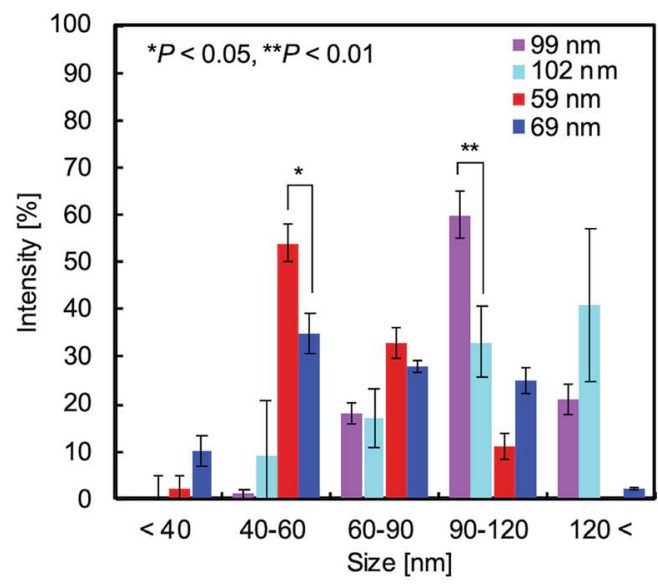

(D)

${ }^{* *} P<0.01,{ }^{* * *} P<0.001$

N.S.: not significant $(P>0.01)$ \#: vs. N.C.

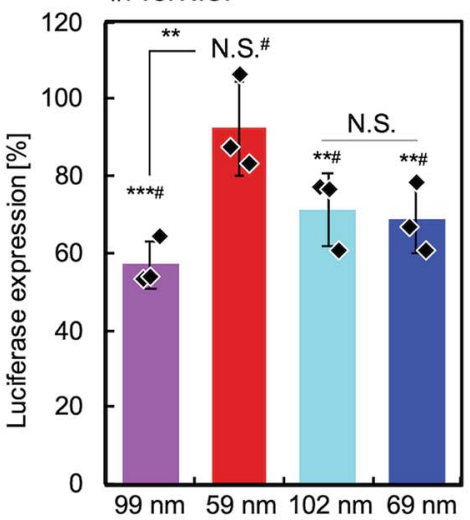

(E)

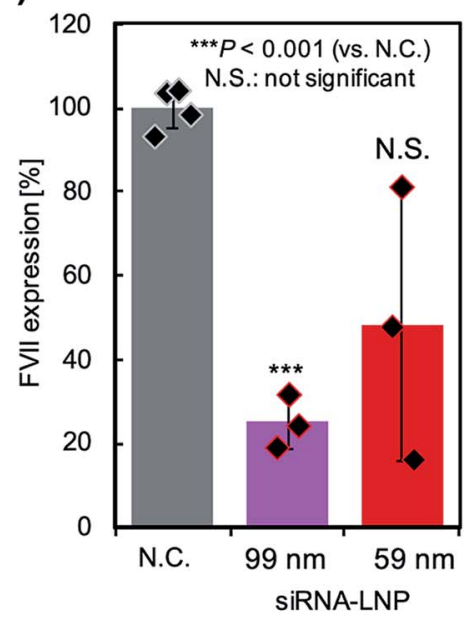

(F)

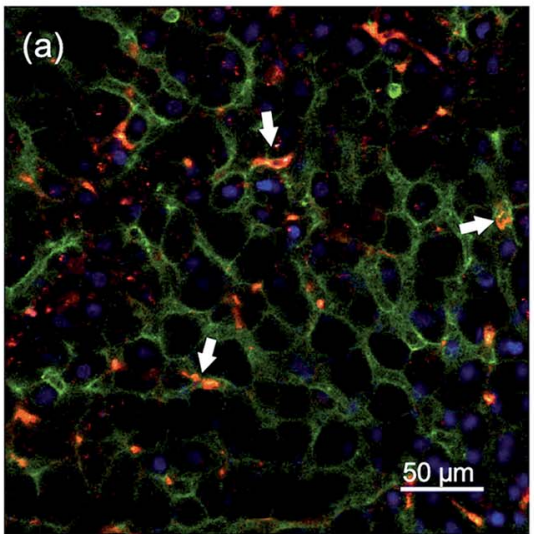

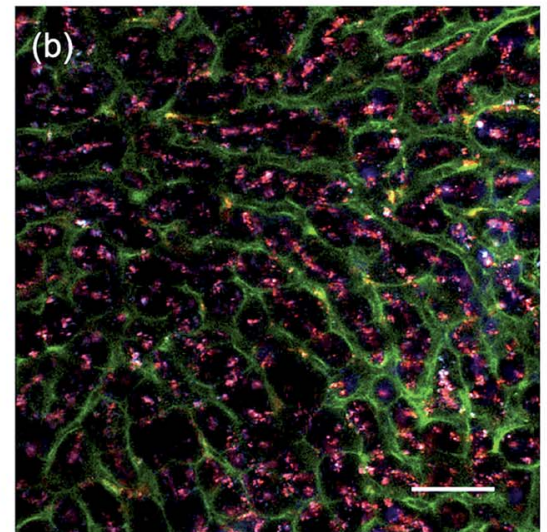

Fig. 6 (A) Schematic of the CL4H6-based LNP preparation and in vivo experimental design. (B) Average size of the CL4H6 LNPs prepared with the 3D and 2D-iLiNP devices. ${ }^{*} * P<0.01$, N.S.: not significant $(P>0.05), n=3$, Student's $t$-test. (C) Size distributions of the CL4H6 LNPs. $* P<0.05$, $* * P<0.01, n=3$, Student's $t$-test. (D) Gene-silencing activity of the 99 and $59 \mathrm{~nm}$ LNPs in HeLa-dluc cells at a dose of $0.1 \mathrm{nM}$ siGL4. $* * P<0.01$, $* * * P<0.001$ (\#: vs. N.C.), N.S.: not significant (\#: vs. N.C., $P>0.01$ ), $n=3$, Student's $t$-test. (E) FVII expression of the ICR mice treated with CL4H6 LNPs at a dose of $0.1 \mathrm{mg} \mathrm{siRNA/kg.}{ }^{* * *} P<0.001$ (vs. N.C.), N.S. not significant $(P>0.01), n=3-4$, Student's $t$-test. (F) Confocal microscope images of the mice liver tissues treated with (a) 99 and (b) $59 \mathrm{~nm}$ LNPs at a dose of $0.5 \mathrm{mg}$ siRNA per kg. Blue, green, red, and cyan represent nuclei, blood vessel, LNP, and siRNA, respectively. The arrows indicate the accumulation of LNPs in the blood vessels. 
intensity of the main peaks of both LNPs at the two size ranges were higher than 50\%. In contrast, 40-60 and >120 nm were the main size ranges of the 69 and $102 \mathrm{~nm}$ LNPs, respectively; however, the intensity of the main peaks of both particles at the two size ranges were lower than $50 \%$. In particular, the intensity of the $99 \mathrm{~nm}$ LNPs in the $90-120 \mathrm{~nm}$ size range was twice as high as that of the 102 nm LNPs.

Next, we carried out an in vitro experiment to evaluate the gene silencing activity of the LNPs with four sizes (Fig. 6(D)). The $99 \mathrm{~nm}$ LNPs showed the best gene silencing activity among the four LNP sizes and suppressed almost $40 \%$ of the luciferase expression at a dose of $0.1 \mathrm{nM}$ siRNA. Interestingly, the 102 and $69 \mathrm{~nm}$ LNPs prepared with the 2D-iLiNP device suppressed luciferase expression by $30 \%$, whereas the $59 \mathrm{~nm}$ LNPs prepared with the 3D-iLiNP device did not induce gene silencing. It was found that gene silencing activity depends on the amount of LNPs with size ranging from 90 to $120 \mathrm{~nm}$. The DODAP LNPs showed a similar trend to that of the CL4H6 LNPs (Fig. S6 $\dagger$ ). This result indicates that the LNP size distribution plays a crucial role on the gene silencing activity, and 3D-iLiNP allows the production of LNPs highly efficient for siRNA delivery. Fig. 6(E) shows the gene silencing activity observed in the in vivo experiment. The lipid system was optimized for the in vivo experiment; ${ }^{19}$ therefore, both the 99 and $59 \mathrm{~nm}$ LNPs suppressed plasma coagulation factor VII (FVII) expression in mice at a dose of $0.1 \mathrm{mg}$ siRNA per $\mathrm{kg}$. The $99 \mathrm{~nm}$ LNPs showed 75\% gene silencing activity, while the $59 \mathrm{~nm}$ LNPs suppressed 50\% of the FVII expression. Fig. 6 (F) shows the intrahepatic LNP distributions of the 99 and $59 \mathrm{~nm}$ LNPs. We observed a sizespecific intrahepatic LNP distribution consistent with previous reports. ${ }^{3}$ From the results of the gene silencing activity experiments, intrahepatic distribution, and size of fenestrae (approximately 100-150 $\mathrm{nm}$ ), the LNPs with a size range of 90$120 \mathrm{~nm}$ exhibited the highest RNA delivery performance in vivo using this lipid system. Therefore, the 3D-iLiNP device will be a key technology for the production of size-controlled LNPs for RNA delivery applications.

\section{Conclusion}

We developed a 3D, symmetrically assembled microfluidic device named 3D-iLiNP with the aim of improving the size distribution of large-sized LNPs produced at low flow rate conditions. The 3D-iLiNP device allowed the production of LNPs with a narrow size distribution owing to the 3D secondary flow. We demonstrated that the 3D-iLiNP device could produce POPC LNPs in the size range of 20 to $100 \mathrm{~nm}$ depending on the flow conditions with $\mathrm{CV}$ values smaller than $10 \%$. We employed the 3D-iLiNP device for the production of siRNA-loaded LNPs to investigate the effect of the LNP size distribution on the gene silencing activity. The 3D-iLiNP device allowed the production of siRNA-loaded LNPs with a size distribution more homogenous than that of the LNPs produced with the 2D-iLiNP device. We clearly distinguished the main LNP size ranges produced by the $3 \mathrm{D}$-iLiNP device and found that the 90-120 nm LNPs suppressed the luciferase expression of HeLa cells. For the in vivo experiment, $100 \mathrm{~nm}$
LNPs showed a higher gene silencing activity than the smallsized siRNA LNPs. We believe that the 3D-iLiNP device and size-controlled LNPs could improve not only the RNA delivery and gene silencing activity, but also the transfection or genome-editing performance of LNP-DDSs.

\section{Experimental procedures}

\section{Materials}

1-Palmitoyl-2-oleoyl-sn-glycero-3-phosphocholine (POPC), 1,2distearoyl-sn-glycero-3-phosphocholine (DSPC), 1,2-dioleoyl-snglycero-3-phosphoethanolamine (DOPE), 1,2-dioleoyl-3dimethylammonium-propane (DODAP), and 1,2-dimyristoylrac-glycero-3-methoxypolyethylene glycol-2000 (DMG-PEG2K) were purchased from the NOF Corporation (Tokyo, Japan). Cholesterol was purchased from Sigma-Aldrich (St. Louis, MO, USA). A pH-sensitive cationic lipid, CL4H6, was synthesized in the laboratory as previously described. ${ }^{19}$ Ethanol, sodium chloride, citric acid, 2-morpholinoethanesulfonic acid (MES), monohydrate, and phosphate-buffered saline (PBS) were purchased from FUJIFILM Wako Pure Chemical Corporation (Osaka, Japan). The siFVII and Cy-5-labeled siGL4 were purchased from Hokkaido System Science Co. Ltd. (Sapporo, Japan). Table $\mathrm{S} 4 \uparrow$ shows the sense and antisense strand sequences of siFVII and Cy5-siGL4. Quant-iTT ${ }^{\mathrm{TM}}$ Ribogreen ${ }^{\mathrm{TM}}$ RNA reagent and $1,1^{\prime}$-dioctadecyl-3,3,3', $3^{\prime}$-tetramethylindocarbocyanine perchlorate (DiI) were obtained from Thermo Fisher Scientific (Waltman, MA, USA). SU-8 3050 was obtained from Nippon Kayaku Co., Ltd. (Tokyo, Japan).

\section{Computational fluid dynamics study}

COMSOL Multiphysics 5.2 (COMSOL, Inc., Burlington, MA) was used for a CFD study. The experimental conditions were the same as those reported in a previous study. ${ }^{14}$ The profiles of the ethanol concentration were numerically simulated using $3 \mathrm{D}$ models under both the laminar-flow model (no slip) and transport of diluted species (ethanol) condition. The flow was modeled as an incompressible flow using the Navier-Stokes equation. The total flow rate and FRR (water/ethanol) were set to $50 \mu \mathrm{L} \min ^{-1}$ and 3 , respectively. For the calculation of the ethanol dilution efficiency, we counted a number of mesh with $20 \%$ ethanol concentration at each cross-section and each residence time. ${ }^{20}$ Then, the ethanol dilution efficiency was calculated from the following equation.

Ethanol dilution efficiency [\%] = number of meshes with $20 \%$ ethanol concentration/total number of meshes $\times 100$

\section{Fabrication of iLiNP devices}

We made poly(dimethylsiloxane) (PDMS) replicas for the 2Dand 3D-iLiNP devices using the standard photolithography method. ${ }^{21}$ For the alignment of the 3D-iLiNP device, we employed amino silane coupling with minor modifications (Fig. $\mathrm{S} 1 \dagger)^{22}$ 


\section{Preparation of LNPs using the 3D-iLiNP device}

POPC was used as a model LNP to investigate the size controllability of the 3D-iLiNP device. POPC LNPs were prepared using a 13.4 mM POPC/ethanol solution and saline. The collected LNP suspensions were dialyzed against saline overnight.

To validate the applicability of the $3 \mathrm{D}$-iLiNP device for the production of siRNA-loaded LNPs, we investigated DODAP- and CL4H6-based LNP systems. The lipid amine-to-oligonucleotide phosphate (N/P) ratio was fixed to 4 . The siRNA-loaded DODAP LNPs were prepared by mixing an $8 \mathrm{mM}$ lipid/ethanol solution composed of DODAP/DOPE/cholesterol/DMG-PEG $2 \mathrm{~K}$ $(30 / 25 / 40 / 5 \mathrm{~mol} \%)$ with a $10 \mathrm{mM}$ citrate buffered solution (pH3.0) containing siRNAs at a concentration of $47.5 \mu \mathrm{g} \mathrm{mL}{ }^{-1}$. The siRNA-loaded CL4H6 LNPs were prepared by mixing a $10 \mathrm{mM}$ lipid/ethanol solution composed of CL4H6/DSPC/ cholesterol/DMG-PEG $2 \mathrm{~K} \quad(50 / 10 / 38.5 / 1.5 \quad \text { mol\% })^{23} \quad$ with a $10 \mathrm{mM}$ citrate buffered solution containing siRNAs at a concentration of $132 \mu \mathrm{g} \mathrm{mL}{ }^{-1}$. The lipid solution and siRNA in the buffer solution were separately introduced into the iLiNP devices at a total flow rates of 50 or $500 \mu \mathrm{L} \mathrm{min} \mathrm{min}^{-1}$ and FRR of 3 . The collected LNP suspensions were dialyzed against $20 \mathrm{mM}$ MES buffer solution ( $\mathrm{pH}$ 6.0) for $2 \mathrm{~h}$ followed by an overnight dialysis against PBS. The siRNA encapsulation efficiency was measured using a Ribogreen assay. ${ }^{3}$ For in vivo experiments, the CL4H6-LNP suspensions were concentrated using Amicon Ultra filters (100K, Merck, Darmstadt, Germany).

The size and polydispersity index (PDI) of the LNPs were measured by dynamic light scattering (DLS) measurements obtained with a Zetasizer Nano ZEN3600 (Malvern Instruments, Worcestershire, U.K.).

\section{In vitro assay}

HeLa cells stably expressing Firefly and Renilla luciferase (HeLa-dluc) were cultured in the same conditions as described previously. ${ }^{19}$ Cells were seeded at a concentration of $3 \times 10^{3}$ and $6 \times 10^{3}$ cells per well in a 96 well-microplate for $24 \mathrm{~h}$ prior to the LNP treatment for the cell viability assay and gene expression assay. Then, the cells were treated with LNPs and incubated for $24 \mathrm{~h}$. After the incubation, the cell viability was measured using a Cell Counting Kit-8 (Dojindo, Kumamoto, Japan) according to the manufacture's protocol. For gene expression assay, firefly and renilla luciferase activities were measured using a Dual-Glo assay and a GloMax®Explorer System (Promega), according to the manufacture's protocol.

\section{In vivo experiment}

The gene knockdown activity of coagulation factor VII (FVII) and the confocal microscope images of the mice's hepatocytes were analyzed following previously described protocols ${ }^{\mathbf{1 4}}$ with minor modifications. For the observation of the intrahepatic siRNA distributions, we prepared DiI- and (Cy5)-labeled siGL4 encapsulated CL4H6 LNPs by mixing a $10 \mathrm{mM}$ lipid/ethanol solution containing $0.5 \mathrm{~mol} \%$ DiI with a $10 \mathrm{mM}$ citrate buffered solution containing Cy5-labeled siGL4. All animal procedures were performed in accordance with the Guidelines for Care and Use of
Laboratory Animals of Hokkaido University and approved by the Hokkaido University Animal Ethics Committee.

\section{Evaluation of scale-up performance of the 3D-iLiNP device}

The 3D-iLiNP device used for the investigation of the massproduced LNPs was made from cycloolefin polymer plates fabricated by a micromachining process (Zeon Corporation, Tokyo, Japan).

\section{Statistical analysis}

The results were analyzed with Microsoft ${ }^{\circledR}$ Excel and an unpaired Student's $t$-test was used to compare the average values of two groups.

\section{Author contributions}

Conceptualization: M. M. and M. T.; data curation: N. K. and M. M.; formal analysis: N. K., M. M., Y. S, A. I., H. T., H. H., and M. T.; methodology: M. M. and M. T.; investigation: N. K., M. M., K. S., and Y. S.; writing-original draft: N. K. and M. M.; writing-review and editing: M. M. and M. T.; funding acquisition: N. K., M. M., H. H., and M. T.; resources: N. K., M. M., and Y. S.; supervision: M. M. and M. T.

\section{Conflicts of interest}

The authors declare no competing financial interest.

\section{Acknowledgements}

This work was supported by JST, CREST Grant Number JPMJCR17H1, Japan, JST, PRESTO Grant Number JPMJPR19K8, Japan, the Special Education and Research Expenses from the Ministry of Education, Culture, Sports, Science and Technology, Supporting Industry Program from the Small and Medium Enterprise Agency, JKA and its promotion funds from KEIRIN RACE, 2020 Feasibility Study Program of the Frontier Chemistry Center, Faculty of Engineering, Hokkaido University, JSPS KAKENHI Grant Number JP19J20939 and JP19KK0140, and Hosokawa Powder Technology Foundation. We would like to thank Editage (www.editage.com) for English language editing.

\section{References}

1 R. A. Mukhamadiyarov, E. A. Senokosova, S. S. Krutitsky, D. V. Voevoda, I. A. Pyshnaya, V. V. Ivanov, M. J. Lewis and I. Khaliulin, J. Cardiovasc. Pharmacol., 2018, 72, 143-152.

2 H. Cabral, Y. Matsumoto, K. Mizuno, Q. Chen, M. Murakami, M. Kimura, Y. Terada, M. R. Kano, K. Miyazono, M. Uesaka, N. Nishiyama and K. Kataoka, Nat. Nanotechnol., 2011, 6, 815-823.

3 Y. Sato, Y. Note, M. Maeki, N. Kaji, Y. Baba, M. Tokeshi and H. Harashima, J. Controlled Release, 2016, 229, 48-57.

4 S. Hirsjarvi, L. Sancey, S. Dufort, C. Belloche, C. VanpouilleBox, E. Garcion, J. L. Coll, F. Hindre and J. P. Benoit, Int. J. Pharm., 2013, 453, 594-600. 
5 H. Ren, Y. He, J. Liang, Z. Cheng, M. Zhang, Y. Zhu, C. Hong, J. Qin, X. Xu and J. Wang, ACS Appl. Mater. Interfaces, 2019, 11, 20304-20315.

6 S. Chen, Y. Y. C. Tam, P. J. C. Lin, M. M. H. Sung, Y. K. Tam and P. R. Cullis, J. Controlled Release, 2016, 235, 236-244.

7 M. Yanez Arteta, T. Kjellman, S. Bartesaghi, S. Wallin, X. Wu, A. J. Kvist, A. Dabkowska, N. Szekely, A. Radulescu, J. Bergenholtz and L. Lindfors, Proc. Natl. Acad. Sci. U. S. A., 2018, 115, E3351-E3360.

8 J. Snoeys, J. Lievens, E. Wisse, F. Jacobs, H. Duimel, D. Collen, P. Frederik and B. De Geest, Gene Ther., 2007, 14, 604-612.

9 S. G. Ong, M. Chitneni, K. S. Lee, L. C. Ming and K. H. Yuen, Pharmaceutics, 2016, 8, 36.

10 A. Jahn, S. M. Stavis, J. S. Hong, W. N. Vreeland, D. L. DeVoe and M. Gaitan, ACS Nano, 2010, 4, 2077-2087.

11 E. Kastner, R. Kaur, D. Lowry, B. Moghaddam, A. Wilkinson and Y. Perrie, Int. J. Pharm., 2014, 477, 361-368.

12 N. Kimura, M. Maeki and M. Tokeshi, in Microfluidics for Pharmaceutical Applications: From Nano/Micro Systems Fabrication to Controlled Drug Delivery, ed. H. A. Santos, D. Liu and H. Zhang, Elsevier, 2019, ch. 5, pp. 123-136.

13 N. Kimura, M. Maeki, Y. Sato, A. Ishida, H. Tani, H. Harashima and M. Tokeshi, ACS Appl. Mater. Interfaces, 2020, 12, 34011-34020.
14 N. Kimura, M. Maeki, Y. Sato, Y. Note, A. Ishida, H. Tani, H. Harashima and M. Tokeshi, ACS Omega, 2018, 3, 50445051.

15 M. Maeki, Y. Fujishima, Y. Sato, T. Yasui, N. Kaji, A. Ishida, H. Tani, Y. Baba, H. Harashima and M. Tokeshi, PLoS One, 2017, 12, e0187962.

16 I. V. Zhigaltsev, N. Belliveau, I. Hafez, A. K. K. Leung, J. Huft, C. Hansen and P. R. Cullis, Langmuir, 2012, 28, 3633-3640.

17 M. Mijajlovic, D. Wright, V. Zivkovic, J. X. Bi and M. J. Biggs, Colloids Surf., B, 2013, 104, 276-281.

18 A. D. Stroock, S. K. Dertinger, A. Ajdari, I. Mezic, H. A. Stone and G. M. Whitesides, Science, 2002, 295, 647-651.

19 Y. Sato, K. Hashiba, K. Sasaki, M. Maeki, M. Tokeshi and H. Harashima, J. Controlled Release, 2019, 295, 140-152.

20 T. J. Johnson, D. Ross and L. E. Locascio, Anal. Chem., 2002, 74, 45.

21 J. C. McDonald and G. M. Whitesides, Acc. Chem. Res., 2002, 35, 491-499.

22 L. Tang and N. Y. Lee, Lab Chip, 2010, 10, 1274-1280.

23 M. Jayaraman, S. M. Ansell, B. L. Mui, Y. K. Tam, J. Chen, X. Du, D. Butler, L. Eltepu, S. Matsuda, J. K. Narayanannair, K. G. Rajeev, I. M. Hafez, A. Akinc, M. A. Maier, M. A. Tracy, P. R. Cullis, T. D. Madden, M. Manoharan and M. J. Hope, Angew. Chem., Int. Ed., 2012, 51, 8529-8533. 